\title{
Optimized Reflector Stacks for Solidly Mounted Bulk Acoustic Wave Resonators
}

\author{
Sumy Jose, André B. M. Jansman, Raymond J. E. Hueting, Senior Member, IEEE, \\ and Jurriaan Schmitz, Senior Member, IEEE
}

\begin{abstract}
The quality factor $(Q)$ of a solidly mounted bulk acoustic wave resonator (SMR) is limited by substrate losses, because the acoustic mirror is traditionally optimized to reflect longitudinal waves only. We propose two different design approaches derived from optics to tailor the acoustic mirror for effective reflection of both longitudinal and shear waves. The first one employs the stopband theory in optics; the second one takes advantage of the periodic nature of reflection spectra in a Bragg reflector: the diffraction grating design approach. The optimized design using stopband theory reaches a calculated minimum transmission of $-25 \mathrm{~dB}$ and $-20 \mathrm{~dB}$ at resonance frequency for longitudinal and shear waves, respectively, for various practical reflector material combinations. Using the diffraction grating approach, a near quarter-wave performance is maintained for longitudinal waves, whereas shear waves reach minimum transmission below $-26 \mathrm{~dB}$. However, this design does necessitate relatively thick layers. The experimental results show good agreement with finite element models (FEM). The extracted 1-D $Q$ for the realized shear optimized devices was increased to around 3300 .
\end{abstract}

\section{INTRODUCTION}

$\mathrm{B}$ ULK-ACOUSTIC-WAVE (BAW) devices are receiving great interest for RF selectivity in mobile communication systems and other wireless applications. Thinfilm BAW devices have several advantages compared with SAW resonators because they are remarkably small in size, and have better power handling abilities and better temperature coefficients, leading to more stable operation [1]. From a practical point of view, SAW filters have considerable drawbacks at frequencies beyond $2 \mathrm{GHz}$, whereas BAW devices up to $20 \mathrm{GHz}$ have been demonstrated [2]. BAW is expected to supersede SAW as the technology of choice in many applications over the next few years as they have now evolved in performance beyond SAW and can be manufactured in a very cost-competitive way using standard IC technology.

BAW technology is commercially available for applications using the U.S. Personal Communication Service (USPCS) (1.85 to $1.91 \mathrm{GHz}$ ) standard. Transmit and receive bands of the US-PCS standard are close in frequency [3]. This demands that the BAW resonators which constitute the narrow band filters must be nearly loss-free. Hence,

Manuscript received May 7, 2010; accepted August 10, 2010. This work was supported by the Dutch Ministry of Economic affairs in the framework of point-one project MEMSLand.

S. Jose, R. J. E. Hueting, and J. Schmitz are with MESA+ Institute for Nanotechnology, Semiconductor Components, University of Twente, The Netherlands (e-mail: s.jose@utwente.nl).

A. B. M. Jansman is with NXP Research, NXP Semiconductors, Eindhoven, The Netherlands.

Digital Object Identifier 10.1109/TUFFC.2010.1749 one of the important goals of BAW community is to come up with high- $Q$ resonators for RF filters by minimizing the losses [4]-[8].

The quality factor $(Q)$ of traditional solidly mounted bulk acoustic wave resonators (SMRs) is traditionally limited by substrate losses [9]-[13], because the conventional quarter-wave acoustic mirror employed in SMRs reflects only the longitudinal acoustic waves and not the shear waves. To obtain high- $Q$ SMRs, the mirror should effectively reflect both of the waves. Therefore, the influence of shear waves on $Q$ was reviewed earlier [11]. Some optimized stacks have been reported for specific material combinations [9], [10] based on numerical calculations.

Recently [12], we reported a systematic design procedure based on a solid theoretical background, derived from optics [14], to design stacks that effectively reflect both longitudinal and shear waves. The salient feature of this method is that it can be applied for any material combination. In this work, we present a more general optimization scheme and also propose an alternative approach based on the periodic nature of reflection spectra of a Bragg reflector. Finite element modeling (FEM) tools have been used to verify the results. In addition, BAW resonators containing the optimized mirror stack have been experimentally characterized showing a considerable improvement in $Q$ compared with devices containing a conventional quarterwavelength stack.

\section{BACKGROUND}

The design of the acoustic mirror is critical in the performance of SMRs. Fig. 1 shows a typical SMR structure in which the piezoelectric resonator is isolated from the substrate using an acoustic mirror [4]. Conventional (quarter-wavelength) mirrors were optimized to reflect only longitudinal acoustic waves. As reported previously [5], the best reflection is obtained if the mirror or reflector consists of alternating layers of low and high acoustic impedances, and that the ratio of these impedances is maximized. For optimal reflection, the layer thickness $t_{n}$ of each layer $n$ is a quarter of the wavelength for longitudinal waves $\lambda_{\text {long }}$ at resonance frequency $f_{\mathrm{R}}$ :

$$
t_{n}=\frac{\lambda_{\text {long }}}{4} .
$$

However, in the quarter-wave mirror designed using (1), shear waves are not optimally reflected. This can be 


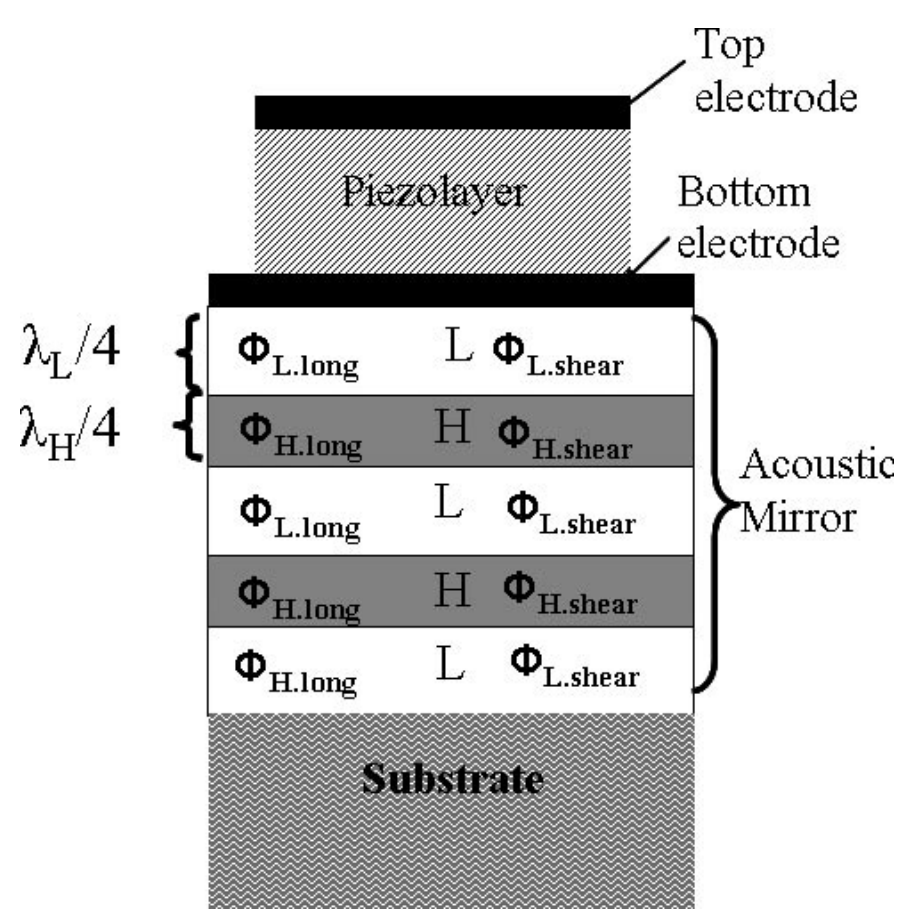

Fig. 1. A schematic cross-section of a solidly mounted BAW resonator (SMR) with a quarter-wave thick acoustic mirror. L and $\mathrm{H}$ indicate layers having low and high acoustical impedance, respectively.

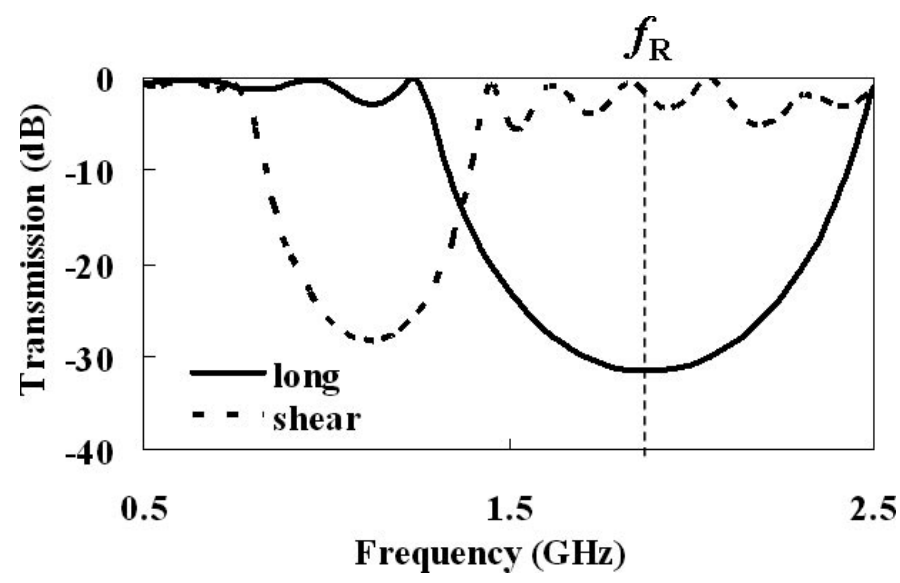

Fig. 2. Calculated transmission curves of longitudinal and shear waves of a nine-layer $\mathrm{Ta}_{2} \mathrm{O}_{5} / \mathrm{SiO}_{2}$ quarter-wave reflector stack optimized for minimum transmission for longitudinal waves at the resonance frequency $f_{\mathrm{R}}=1.88 \mathrm{GHz}$. Longitudinal transmission at $f_{\mathrm{R}}$ is $-31.5 \mathrm{~dB}$ and shear transmission at $f_{\mathrm{R}}$ is $-0.59 \mathrm{~dB}$.

explained by the fact that the shear wave velocity is typically about half of the longitudinal wave velocity [9]. By implementing a transmission line model, and with the approach of mirror reflectivity [13] but with the top-most mirror layer as the reference point, the transmission curve as a function of frequency of such a mirror is computed in MathCAD (Parametric Technology Corp., Needham, MA) for the mirror stack. Fig. 2 shows the transmission curves of a nine-layer $\mathrm{SiO}_{2} / \mathrm{Ta}_{2} \mathrm{O}_{5}(762 \mathrm{~nm} / 588 \mathrm{~nm})$ quarter-wave mirror optimized at $f_{\mathrm{R}}=1.88 \mathrm{GHz}$ for minimum transmission for longitudinal waves. It can be seen that the minimum transmission of longitudinal waves corre- sponds to maximum transmission for shear waves. This emphasizes that dual reflection in this particular stack is not possible. One possible solution to obtain dual reflection would be employing two sets of stacks; one reflecting longitudinal and another set reflecting shear waves. However, this would increase the number of layers and, hence, the fabrication complexity. In Section III, we present some design approaches derived from optics to solve this problem.

\section{Design Approaches}

Two design approaches are discussed in this section. Section III-A describes an approach to modify the stacks as proposed recently [12], but extended with a more general optimization scheme. An alternative approach to modify the stacks taking advantage of the periodic nature of the reflection spectra of the Bragg reflector is proposed in Section III-B. Note that the translation from optics to acoustics is an important issue. For the BAW filters, we need to reflect longitudinal and shear acoustic waves at the same $f_{\mathrm{R}}$, but each has a different wave velocity, whereas in optical filters the opposite would be the case.

\section{A. The Stopband Theory Design Approach}

It was as early as 1917 that Lord Rayleigh [15] studied the natural stratification in some insects which are periodic structures of alternative high and low refractive indices. It was perhaps here that the first stopband theory was defined. It was later redeveloped by various authors in various terms to study optics in thin film dielectric periodic structures [14]. Presently the theory is well defined in optics of thin films. Here, we further elaborate on the original stopband theory approach used for acoustic waves described recently [12].

For the simplest periodic mirror structure, the basic unit consists of two dielectric layers as shown in Fig. 1 (dashed lines) with high $(\mathrm{H})$ and low $(\mathrm{L})$ refractive indices. The optics of thin films defines a stopband as being a high-reflection region (or equivalently a low-transmission region) which is a characteristic feature of any periodic structure. The cardinal point of the stopband is defined as the point of maximum reflection (minimum transmission) in this region. The well-known quarter-wavelength stack (denoted as LH) gives reflection for the frequencies at which the phase drops over the high- and low-index layers are both $\pi / 2$. One can deviate from these equal phases and still obtain reflection bands. The stopband theory [14] states that an $\mathrm{L}(c \cdot \mathrm{H})$-stack (i.e., a stack consisting of bi-layers where the ratio of phases over the low and high index equals an integer $c$ ) gives a maximum reflection for the frequencies at which the phase drop over L-layer equals one of the so-called cardinal points:

$$
\varphi_{n}=\frac{n \pi}{1+c},
$$


where $n$ takes integer values $n \leq c$ and $c=\phi_{\mathrm{H}} / \phi_{\mathrm{L}} ; \phi_{\mathrm{L}}$ and $\phi_{\mathrm{H}}$ are the phase drops in low-index and high-index layers, respectively. If we choose $\phi_{n}$ as the phase of the low-index layer, the corresponding phase of the high-index layer is $c \cdot \phi_{n}$, with $c$ as the detuning parameter. Note that for $c=$ 1 , the stack reduces to the quarter-wavelength $(\mathrm{LH})$ case.

Strictly speaking, the stopband theory restricts $c$ to be integer numbers. However, one can choose $c$ to be non-integer at the cost of unequal reflection efficiency. Still, the reflection bands appear at frequencies for which the phase over the L-layer equals $n \cdot \pi /(1+c)$. Furthermore, large values of $n$ and $c$ lead to large phase drops over the layers, leading to relatively thick layers. Because this increases fabrication cost, it is undesirable. Therefore, the numbers $c$ and $n$ are kept at a minimum.

Further, for $\phi_{\mathrm{L}}$ and $\phi_{\mathrm{H}}$ it also holds that

$$
\phi_{\mathrm{L}}+\phi_{\mathrm{H}}=n \pi
$$

Hence, (2) and (3) state that [14]:

$$
\phi_{\mathrm{L}}+\phi_{\mathrm{H}}=(1+c) \varphi_{n}=n \pi \text {. }
$$

As mentioned previously, the objective is to design a reflector stack that reflects both longitudinal and shear waves. The stopband theory has given us a degree of freedom: the detuning parameter $c$. Hence, the task is to find thicknesses of the low- and high-index layer, $t_{\mathrm{L}}$ and $t_{\mathrm{H}}$ respectively, such that the sum of phase drops over a bilayer equals a multiple of $\pi$ for both types of waves. In the following sections, we will introduce a basic design method and a more general optimization scheme for an acoustic reflector design.

1) The Basic Reflector Design Approach: Consider an L $(c \cdot \mathrm{H})$ stack, where $c$ is now defined in terms of longitudinal waves:

$$
c=\frac{\phi_{\text {H.long }}}{\phi_{\text {L.long }}} .
$$

At the frequency of maximum reflection, the cardinal points equal the phase drops over the layers $\left(\varphi_{n \cdot \mathrm{L}}=\phi_{\mathrm{L}}\right.$, $\left.\varphi_{n \cdot \mathrm{H}}=\phi_{\mathrm{H}}\right)$, and let's say the longitudinal waves will have reflection bands at the frequency for which the phase drop over the L-layer, $\phi_{\text {L.long }}$ is:

$$
\phi_{\mathrm{L} . l o n g}=\frac{n \pi}{1+c}
$$

From (5) and (6), we can find the phase drop over the H-layer as:

$$
\phi_{\mathrm{H} . l o n g}=\frac{n c \pi}{1+c} \text {. }
$$

Setting this frequency equal to a desired frequency, $f_{\mathrm{R}}$, at which a good reflection is desired, we can calculate the thicknesses of the H- and L-layers as

$$
\begin{aligned}
& t_{\mathrm{L}}=\frac{\phi_{\mathrm{L} . \text { long }}}{2 \pi} \cdot \frac{v_{\mathrm{L} . \text { long }}}{f_{\mathrm{R}}}=\frac{n}{2(1+c)} \lambda_{\mathrm{L} . \text { long }} \\
& t_{\mathrm{H}}=\frac{\phi_{\mathrm{H} . \text { long }}}{2 \pi} \cdot \frac{v_{\mathrm{H} . \text { long }}}{f_{\mathrm{R}}}=\frac{c n}{2(1+c)} \lambda_{\mathrm{H} . \text { long }},
\end{aligned}
$$

where $v_{\mathrm{L} . l o n g}$ and $v_{\mathrm{H} . l o n g}$ are the velocities of the longitudinal waves in the low- and high-acoustic impedance layer, respectively, and $\lambda_{\text {L.long }}$ and $\lambda_{\text {L.long }}$ are the corresponding wavelengths.

The phase drop over these layers for shear waves equals

$$
\phi_{\text {L.shear }}=2 \pi f_{\mathrm{R}} \cdot\left(\frac{t_{\mathrm{L}}}{v_{\text {L.shear }}}\right) \text {. }
$$

Substituting $t_{\mathrm{L}}$ from (8) and simplifying, we obtain

$$
\phi_{\text {L.shear }}=\frac{n \pi}{(1+c)} \cdot\left(\frac{v_{\text {L.long }}}{v_{\text {L.shear }}}\right)=\frac{n \pi}{(1+c)} \cdot K_{\mathrm{L}} .
$$

Similarly, we can find the phase of the shear waves in the $\mathrm{H}$ layer as

$$
\phi_{\mathrm{H} . \text { shear }}=\frac{n \cdot c \pi}{(1+c)} \cdot\left(\frac{v_{\text {H.long }}}{v_{\text {H.shear }}}\right)=\frac{n \cdot c \pi}{(1+c)} \cdot K_{\mathrm{H}},
$$

where $v_{\text {L.shear }}$ and $v_{\text {H.shear }}$ are the velocity of shear waves in the low- and high-impedance layers, respectively, and $K_{\mathrm{L}}$ $=v_{\text {L.long }} / v_{\text {L.shear }}, K_{\mathrm{H}}=v_{\text {H.long }} / v_{\text {H.shear }}$.

In general, the sum of the phases of shear waves in $\mathrm{L}$ and $\mathrm{H}$ layers $[(10)+(11)]$ will result in $n \pi$. For most of the practical materials, the ratios $K_{\mathrm{L}}$ and $K_{\mathrm{H}}$ are not far from 2. Hence, if the sum of phases for the longitudinal wave is $n \pi$, then the sum of phases for the shear waves will be close to $2 \mathrm{n} \pi$, which also follows (3). With this approximation, we can calculate the thicknesses of the $\mathrm{L}$ and $\mathrm{H}$ layers that can reflect both the waves. Acknowledging that the longitudinal wave is the primary wave in a BAW resonator carrying the most acoustic energy, we emphasize our optimization to this type of wave. Therefore, we set the detuning parameter $c$ for longitudinal waves equal to an integer number. The quarter wave stack $(c=1, n=1$ for longitudinal waves) gives $c=1, n=2$ for shear waves if the ratios $K_{\mathrm{L}}$ and $K_{\mathrm{H}}$ equal 2 . This is not a reflection band, because $n$ should satisfy the condition $n \leq c$. Next is $c=2, n=1$ for the longitudinal wave. This gives $c=$ $2, n=2$ for the shear wave, fulfilling all the conditions of a cardinal point. To conclude, the $\mathrm{L} 2 \mathrm{H}$ configuration can simultaneously reflect both longitudinal and shear waves.

The thicknesses given by (8) provide maximum reflection for both longitudinal and shear waves at a particular frequency, $1.88 \mathrm{GHz}$. This zeroth-order method often leads to satisfactory results if $K_{\mathrm{L}}$ and $K_{\mathrm{H}}$ are not too far from 2 for both materials. Also for cases where the average of $K_{\mathrm{L}}$ and $K_{\mathrm{H}}$ is around 2 , reasonable reflection coefficients can be expected. It is inferred from the simulations that to obtain optimum results for dual reflection of longitudinal 


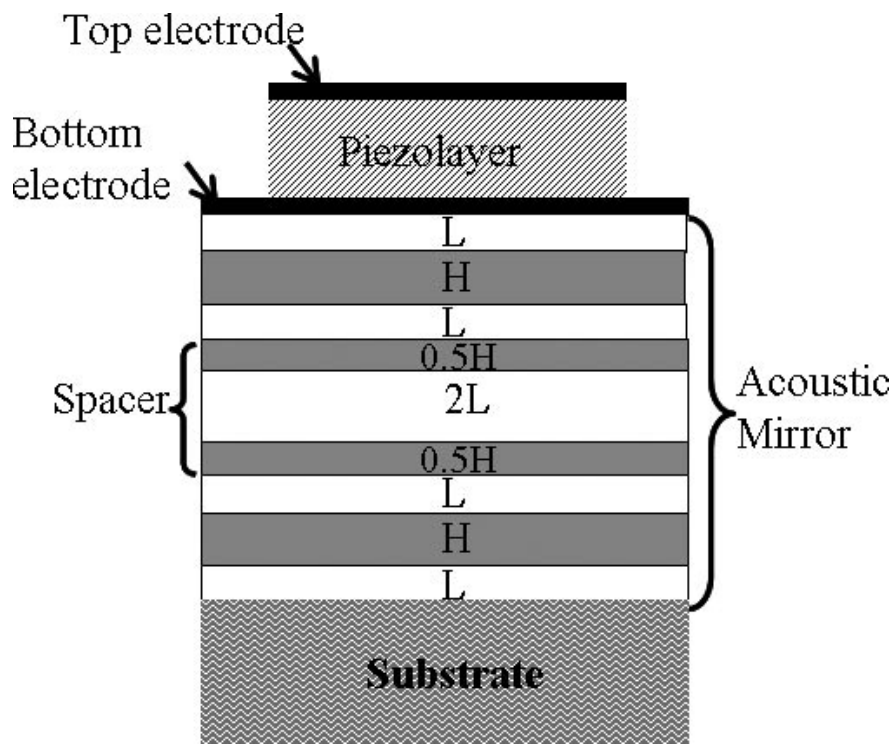

Fig. 3. Improved layer configuration with the optimized thicknesses for the acoustic mirror of solidly mounted BAW resonator (SMR). The spacer layer is designed according to [13].

and shear waves in low- and high-impedance layers, $c$ can be taken as the average of velocity ratios as

$$
c=\frac{K_{\mathrm{L}}+K_{\mathrm{H}}}{2} \approx 2 \text {. }
$$

These thicknesses can be used in an improved layer configuration by introducing a spacer layer $2 \mathrm{H} 2 \mathrm{~L} 2 \mathrm{H} \mathrm{[16]}$ to further optimize the reflection bands for both types of waves. In optics, it was suggested to tune this spacer layer by using a trial-and-error method to align the edges of the bands and hence improve the performance [17]. However, we optimized the reflector stack, which is actually a Fabry-Perot resonator, using a transmission line model [18]. We observed that a $0.5 \mathrm{H} 2 \mathrm{~L} 0.5 \mathrm{H}$ configuration acts as Fabry-Perot cavity for both longitudinal and shear waves. The improved layer configuration of the acoustic mirror is (LHL) $(0.5 \mathrm{H} 2 \mathrm{~L}$ 0.5H) (LHL) [12] which is shown in Fig. 3 . Note that the numerals in the configuration indicate the relative thickness, e.g., $0.5 \mathrm{H}$ indicates $0.5 \cdot \lambda_{\mathrm{H} . l o n g}$.

The results from the one-dimensional analytical model are presented in Fig. 4. It shows the transmission curve for the optimized reflector stack with and without the spacer. It can be seen from the figure that using the optimized thicknesses calculated by the new design procedure has minimized the transmission at $1.88 \mathrm{GHz}$, and using these thicknesses in the (improved) spacer configuration brings the transmission bands into coincidence. The effect for various material combinations and the dependence of the layer thickness for the spacer configuration were verified earlier [12].

2) A Novel Optimization Scheme: The basic reflector design method gives best results if $K_{\mathrm{L}}$ and $K_{\mathrm{H}}$ are close to 2, which is true for most of the materials [9]. However, in some cases in which the velocity ratio deviates from 2 , the basic stopband design approach becomes less accu-

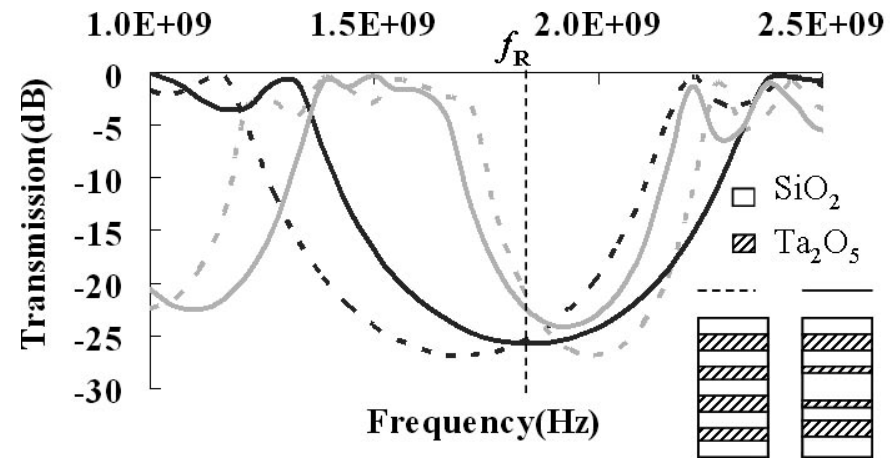

Fig. 4. Transmission curves of a nine-layer $\mathrm{SiO}_{2} / \mathrm{Ta}_{2} \mathrm{O}_{5}$ optimized reflector stack based on the new design procedure with and without the spacer layer. Black lines indicate longitudinal and gray indicate shear waves. Solid lines represent the configuration with the spacer layer and dashed lines represent the ones without the spacer layer. Longitudinal transmission is $-25.7 \mathrm{~dB}$ and shear transmission is $-22.8 \mathrm{~dB}$ at resonance frequency.

rate. In this section, we propose a more general approach for dual reflection by optimizing the so-called phase error. This phase error (or phase offset) is the phase difference between the actual phase at the cardinal points and the calculated phase. The thicknesses are optimized in such a way that the phase offset from the transmission minima (cardinal points) for longitudinal and shear waves are equal, and hence the phase error is minimal in both cases. Defining $\beta_{\mathrm{L}}$ and $\beta_{\mathrm{H}}$ as the wave numbers in low- and highimpedance layers, respectively, the phase drops $\phi_{\mathrm{L}}=\beta_{\mathrm{L}}$. $t_{\mathrm{L}}$ and $\phi_{\mathrm{H}}=\beta_{\mathrm{H}} \cdot t_{\mathrm{H}}$ can be conveniently written in terms of thicknesses and wave velocity as [refer to (3)]

$$
\phi_{\mathrm{L}}+\phi_{\mathrm{H}}=\frac{2 \pi t_{\mathrm{L}}}{\lambda_{\mathrm{L}}}+\frac{2 \pi t_{\mathrm{H}}}{\lambda_{\mathrm{H}}}=2 \pi f_{\mathrm{R}}\left(\frac{t_{\mathrm{L}}}{v_{\mathrm{L}}}+\frac{t_{\mathrm{H}}}{v_{\mathrm{H}}}\right)=n \pi .
$$

In acoustics, $v_{\mathrm{L}}$ and $v_{\mathrm{H}}$ have different values for the case of longitudinal and shear waves. We need to obtain thickness $t_{\mathrm{L}}$ and $t_{\mathrm{H}}$, such that (12) is fulfilled for both longitudinal and shear waves at one frequency $f_{\mathrm{R}}$ :

$$
\begin{aligned}
2 \pi f_{\mathrm{R}}\left(\frac{t_{\mathrm{L}}}{v_{\text {L.long }}}+\frac{t_{\mathrm{H}}}{v_{\text {H.long }}}\right) & =n_{1} \pi, \\
2 \pi f_{\mathrm{R}}\left(\frac{t_{\mathrm{L}}}{v_{\text {L.shear }}}+\frac{t_{\mathrm{H}}}{v_{\text {H.shear }}}\right) & =n_{2} \pi .
\end{aligned}
$$

This set of two equations with two unknowns, $t_{\mathrm{H}}$ and $t_{\mathrm{L}}$, is solvable for all values of positive integers $n_{1}$ and $n_{2}$. Again, the ratio of longitudinal and wave velocities of most materials is around 2. For such values, only nonnegative values for the thicknesses are found if $n_{1}$ and/or $n_{2}$ are larger than 3 . This translates into layer thicknesses well above $1000 \mathrm{~nm}$. In fabrication, this is impractical: such layers take a long time to deposit and their thickness cannot be controlled accurately enough.

Let us allow a phase error $\Delta \phi$ in (14) such that it has the same magnitude for shear and longitudinal waves. To keep the thicknesses of the layers small, we set $n_{1}$ and $n_{2}$ to 1 and 2, respectively, as in a $\mathrm{L} 2 \mathrm{H}$ configuration. 


$$
\begin{gathered}
\phi_{\text {L.long }}+\phi_{\text {H.long }}=\pi+\Delta \phi, \\
\phi_{\text {L.shear }}+\phi_{\text {H.shear }}=2 \pi-\Delta \phi .
\end{gathered}
$$

$\phi_{\text {L.long }}$ and $\phi_{\text {L.shear }}$ are the phase drops over the low-impedance layer for the longitudinal and shear waves at the same frequency $f_{\mathrm{R}}$. Likewise, $\phi_{\mathrm{H} \text {.long }}$ and $\phi_{\mathrm{H} \text {.shear }}$ are the phase drops over the high-impedance layer at this frequency.

Introducing $K_{\mathrm{L}}$ and $K_{\mathrm{H}}$ in (16) gives:

$$
K_{\mathrm{L}} \phi_{\mathrm{L} . \text { long }}+K_{\mathrm{H}} \phi_{\mathrm{H} . \text { long }}=2 \pi-\Delta \phi .
$$

By summing (15) and (17), the phase difference drops out:

$$
\phi_{\mathrm{L} . \mathrm{long}}\left(1+K_{\mathrm{L}}\right)+\phi_{\mathrm{H} . \text { long }}\left(1+K_{\mathrm{H}}\right)=3 \pi .
$$

Now introducing $c$ in (18) gives:

$$
\begin{gathered}
\phi_{\mathrm{L} . \text { long }}\left(1+c+K_{\mathrm{L}}+c K_{\mathrm{H}}\right)=3 \pi . \\
\phi_{\mathrm{L} . \text { long }}=\frac{3 \pi}{1+c+K_{\mathrm{L}}+c K_{\mathrm{H}}}, \\
\phi_{\mathrm{H} . \text { long }}=c \phi_{\mathrm{L} . \text { long }}=\frac{3 \pi c}{1+c+K_{\mathrm{L}}+c K_{\mathrm{H}}} .
\end{gathered}
$$

Then, the associated layer thicknesses are calculated in a straight-forward way:

$$
\begin{aligned}
& t_{\mathrm{L}}=\frac{\phi_{\mathrm{L} . \text { long }}}{2 \pi} \lambda_{\mathrm{L} . \text { long }}=\frac{3 / 2}{1+c+K_{\mathrm{L}}+c K_{\mathrm{H}}} \lambda_{\mathrm{L} . \text { long }} \\
& t_{\mathrm{H}}=\frac{\phi_{\mathrm{H} . \text { long }}}{2 \pi} \lambda_{\mathrm{H} . \text { long }}=\frac{3 c / 2}{1+c+K_{\mathrm{L}}+c K_{\mathrm{H}}} \lambda_{\mathrm{H} . \text { long }}
\end{aligned}
$$

We have come to an expression for the layer thicknesses giving an equal phase error $\Delta \phi$ in (15) and (16). The coefficient $c$ is a degree of freedom that we will use later. Substituting (20) into (15) gives the phase error applicable for both longitudinal and shear waves:

$$
\Delta \phi=\frac{\left(2+2 c-K_{\mathrm{L}}-c K_{\mathrm{H}}\right) \pi}{1+c+K_{\mathrm{L}}+c K_{\mathrm{H}}} .
$$

In summary, a reflector stack can be optimized according to (21). In this case, $c$ is a variable parameter. We have verified the performance of reflectors built according to (21) for various reflector materials, the properties of which are given in Table I. In Fig. 5, we present the transmission plotted against $c$ calculated for reflector stacks with varying number of layers, using different material combinations, demonstrating that good performance is obtained for $1<c<2$. It is seen from Fig. 5 that for each bi-layer, the transmission decreases by a fixed amount for a given material combination and this becomes more and more accurate when there are more reflector layers. A low transmission for longitudinal and shear waves is also obtained for $c$ values around 0.5. Nonetheless, this working
TABle I. Summary of Velocity Ratio Used for Reflectors.

\begin{tabular}{lc}
\hline Material & $K=v_{\text {long }} / v_{\text {shear }}$ \\
\hline $\mathrm{SiO}_{2}$ & 1.64 \\
$\mathrm{Ta}_{2} \mathrm{O}_{5}$ & 1.81 \\
$\mathrm{~W}$ & 1.77 \\
$\mathrm{SiOC}$ & 1.55 \\
$\mathrm{AlN}$ & 1.85 \\
$\mathrm{Pt}$ & 2.43 \\
\hline
\end{tabular}

point is less attractive; for most material combinations the transmission for longitudinal waves is larger than for shear waves. For a high-performance BAW resonator, the transmission of longitudinal waves should be lower than $-35 \mathrm{~dB}$ and for shear waves it should be lower than $-18 \mathrm{~dB}[10]$.

In Fig. 6, we present the transmission curves for ninelayer reflector stacks with thicknesses optimized for the minimum transmission of longitudinal and shear waves at resonance frequency using (21) by varying $c$. The advantage of $c$ being a tunable parameter is that the transmission of longitudinal and shear waves can be improved according to the requirements and hence this method allows the designer to find the trade-off between longitudinal and shear reflection by choosing the proper value for $c$.

The thicknesses obtained with this design approach can also be combined with the spacer layer configuration proposed recently [12] (see Fig. 3) to further bring the bands into coincidence, hence achieving the dual reflection of both waves in the same frequency band. The improvements by the more general optimization scheme over the basic stopband theory approach would be more pronounced if the velocity ratio deviates much from 2 . However, the improvement of the longitudinal wave transmission is always at the cost of shear wave transmission. Therefore, depending on the requirements, a choice should be made between the design approaches. A comparison of longitudinal $\left(T_{\text {long }}\right)$ and shear $\left(T_{\text {shear }}\right)$ transmission coefficients of reflector stack designed using basic stopband theory approach [using (8)] and improvements using the more general optimization scheme [using (21)] are given in Table II.

We have also done an analysis varying the number of layers in the reflector stack, retaining the periodicity of the improved stack configuration. This is shown in Fig. 7. As expected, it is found that a reflector stack with an odd number of layers has a better performance. This holds for any multilayer periodic system [19].

3) The Diffraction Grating Approach: In this section, the problem of dual reflection is solved using the fact that the reflector stack is essentially a periodic structure and hence behaves much like a diffraction grating. Any arrangement which is equivalent in its action to several parallel equidistant slits of the same width is called a diffraction grating [20]. The principle of diffraction grating is to reflect the wave back by introducing some periodicities in the grating, thus cancelling the phase shift upon reflection. Fig. 


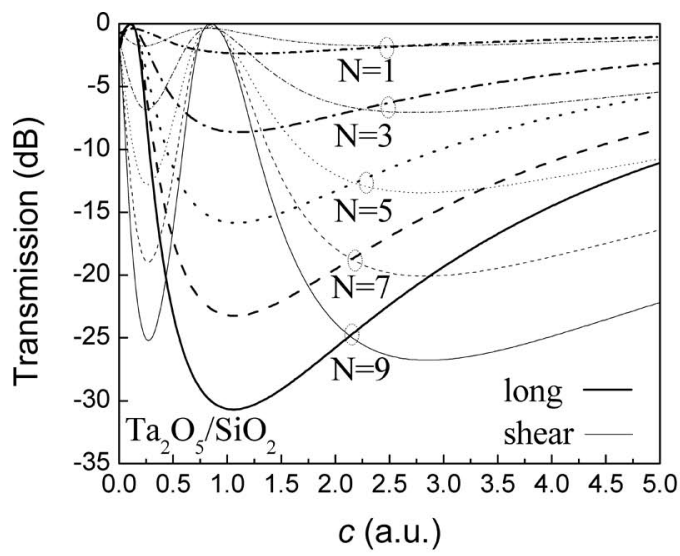

(a)

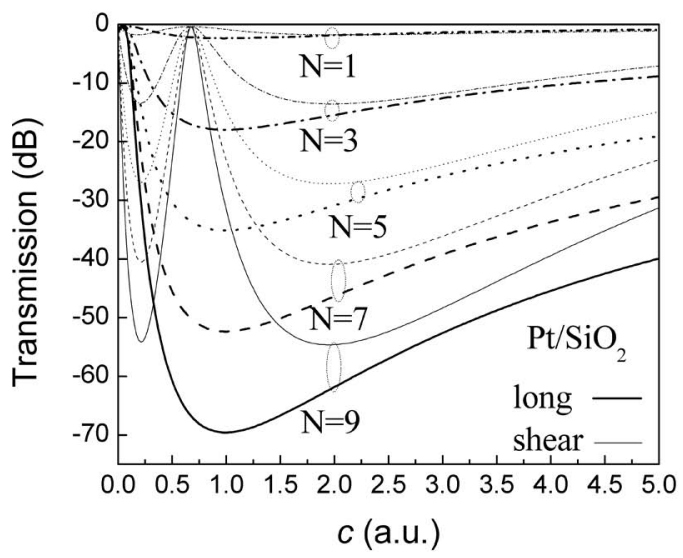

(c)

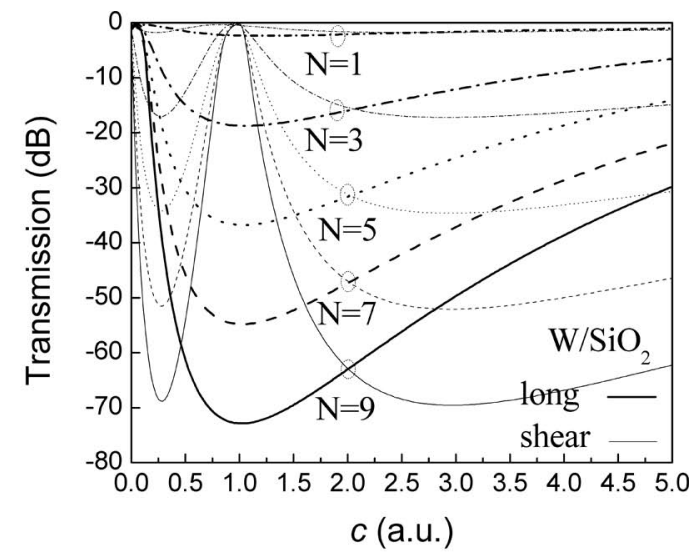

(b)

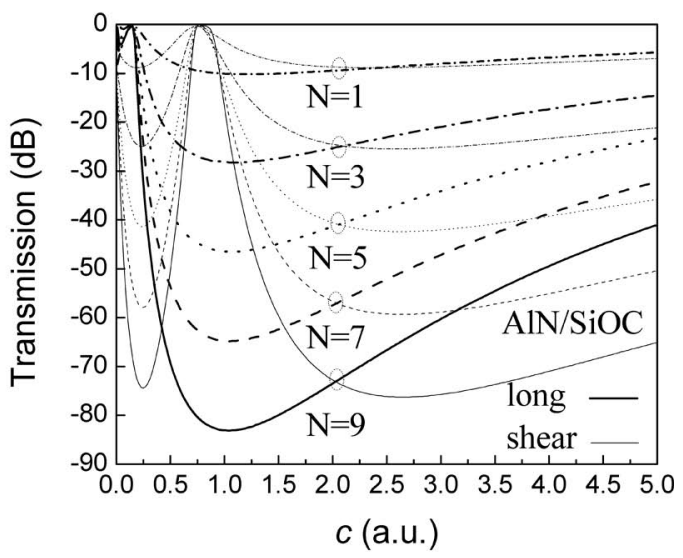

(d)

Fig. 5. Transmission versus the phase ratio $c$ at resonant frequency for reflector stacks with varying number of layers demonstrated for different material combinations: (a) $\mathrm{Ta}_{2} \mathrm{O}_{5} / \mathrm{SiO}_{2}$, (b) $\mathrm{W} / \mathrm{SiO}_{2}$, (c) $\mathrm{Pt} / \mathrm{SiO}_{2}$, (d) AlN/SiOC. In the calculations the mirror stack was loaded with AlN on the top and $\mathrm{Si}$ at the bottom. $\mathrm{N}$ represents the number of layers used for the mirror stack.

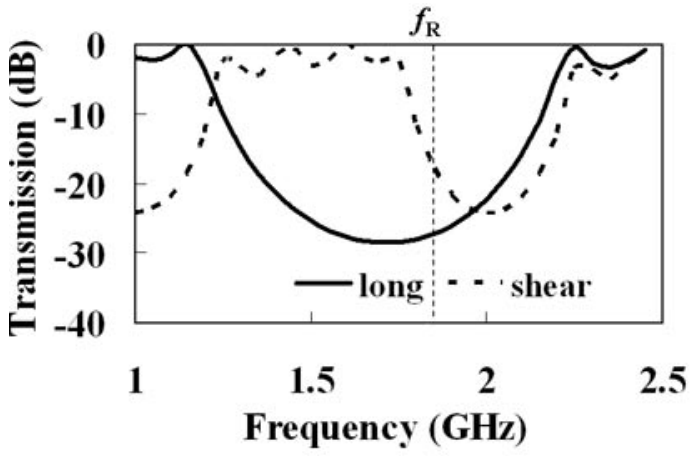

(a)

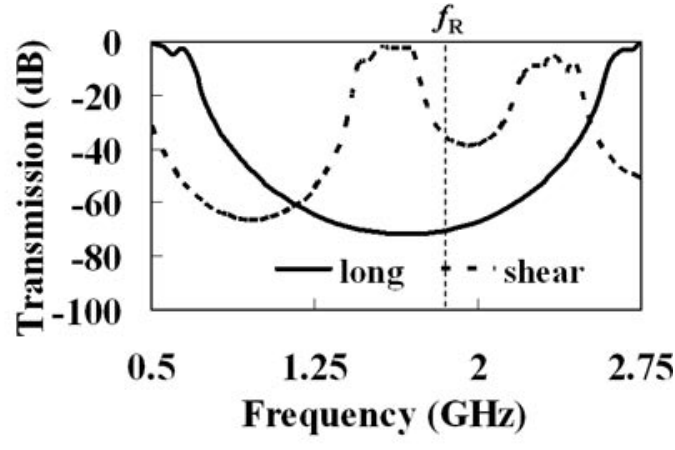

(b)

Fig. 6. Transmission curves for longitudinal and shear waves in a nine-layer reflector stack of (a) $\mathrm{SiO}_{2} / \mathrm{Ta}_{2} \mathrm{O}_{5}(c=1.7)$ and $(\mathrm{b}) \mathrm{SiO}_{2} / \mathrm{W}(c=1.2)$. The solid and dotted lines indicate longitudinal and shear waves, respectively. The vertical dotted lines indicate the frequency of optimization $\left(f_{\mathrm{R}}\right.$ $=1.88 \mathrm{GHz}$ ). The reason for much lower transmission coefficients in (b) compared with (a) is attributed to the high acoustic impedance ratio of $\mathrm{SiO}_{2} / \mathrm{W}$.

TABLE II. The Improvements by the General Stopband Theory Approach.

\begin{tabular}{lccccc}
\hline \multicolumn{1}{c}{ Stack } & $T_{\text {long }}(\mathrm{dB})$ & $T_{\text {shear }}(\mathrm{dB})$ & $c$ & $\begin{array}{c}\text { Optimized } \\
T_{\text {long }}(\mathrm{dB})\end{array}$ & $\begin{array}{c}\text { Optimized } \\
T_{\text {shear }}(\mathrm{dB})\end{array}$ \\
\hline $\mathrm{SiO}_{2} / \mathrm{Ta}_{2} \mathrm{O}_{5}$ & -25.7 & -22.8 & 1.7 & -27.4 & -17.3 \\
$\mathrm{SiO}_{2} / \mathrm{Pt}$ & -60.8 & -53.9 & 1.2 & -67.3 & -47.1 \\
$\mathrm{SiO}_{2} / \mathrm{W}$ & -63.6 & -56.6 & 1.2 & -70.5 & -35.2 \\
\hline
\end{tabular}




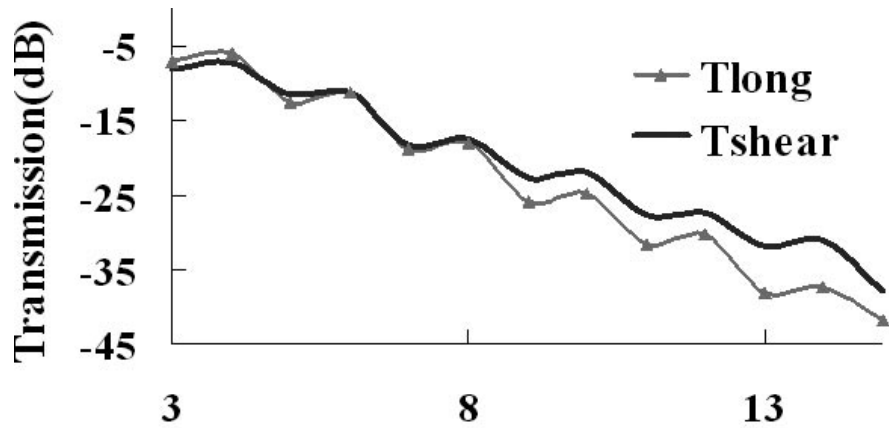

No. of layer stacks

Fig. 7. Transmission versus number of layer stacks in the improved stack configuration.

8 shows the reflection spectra of a nine-layer $\mathrm{Ta}_{2} \mathrm{O}_{5} / \mathrm{SiO}_{2}$ quarter-wave reflector stack. Apart from the first-order reflections at $f_{\mathrm{R}}$ corresponding to $t=\lambda_{\text {long }} / 4$, we can also see higher-order reflections [19], [20] which correspond to the layer thickness:

$$
t=\frac{\lambda_{\text {long }}}{4}+m \cdot \frac{\lambda_{\text {long }}}{2}=(1+2 m) \cdot \frac{v_{\text {long }}}{4 f_{\mathrm{R}}},
$$

where $m$ is a nonnegative integer.

Here, we will make use of these higher-order reflections. By changing the value of $m$ in (23), it is possible to have reflection (for a given $f_{\mathrm{R}}$ ) for waves with velocity $v_{\text {long }}$ at $m=m_{1}$ and for waves with velocity $v_{\text {shear }}$ at $m=m_{2}$ for a particular layer thickness.

Consider a regular $(\mathrm{LH})^{n}$ layer stack. Let us look at a single layer, say a low-impedance layer L having a thickness $t_{\mathrm{L}}$ which corresponds to a quarter wavelength.

Looking for the higher-order reflections in the L layer so that both longitudinal and shear waves propagate, (23) can be written as:

$$
t_{\mathrm{L}}=\frac{\left(1+2 \cdot m_{1}\right) \cdot v_{\mathrm{L} . \text { long }}}{4 f_{\mathrm{R}}}=\frac{\left(1+2 \cdot m_{2}\right) \cdot v_{\mathrm{L} . \text { shear }}}{4 f_{\mathrm{R}}} .
$$

Working out (24) gives:

$$
\frac{\left(1+2 \cdot m_{2}\right)}{\left(1+2 \cdot m_{1}\right)}=\frac{v_{\text {L.long }}}{v_{\text {L.shear }}}=K_{\mathrm{L}}
$$

From (25), it is deduced that two integers $m_{1}$ and $m_{2}$ are to be estimated that should satisfy the condition:

$$
m_{2}=K_{\mathrm{L}} \cdot m_{1}+\frac{\left(K_{\mathrm{L}}-1\right)}{2} .
$$

For the high-impedance layer $\mathrm{H}$, we will have different velocities $v_{\text {H.long }}$ and $v_{\text {H.shear }}$, for which we are able to find different integers $m_{3}$ and $m_{4}$, and the thickness of the $\mathrm{H}$ layer can be written as

$$
t_{\mathrm{H}}=\frac{\left(1+2 \cdot m_{3}\right) \cdot v_{\mathrm{H} . \text { long }}}{4 f_{\mathrm{R}}}=\frac{\left(1+2 \cdot m_{4}\right) \cdot v_{\mathrm{H} . s h e a r}}{4 f_{\mathrm{R}}} .
$$

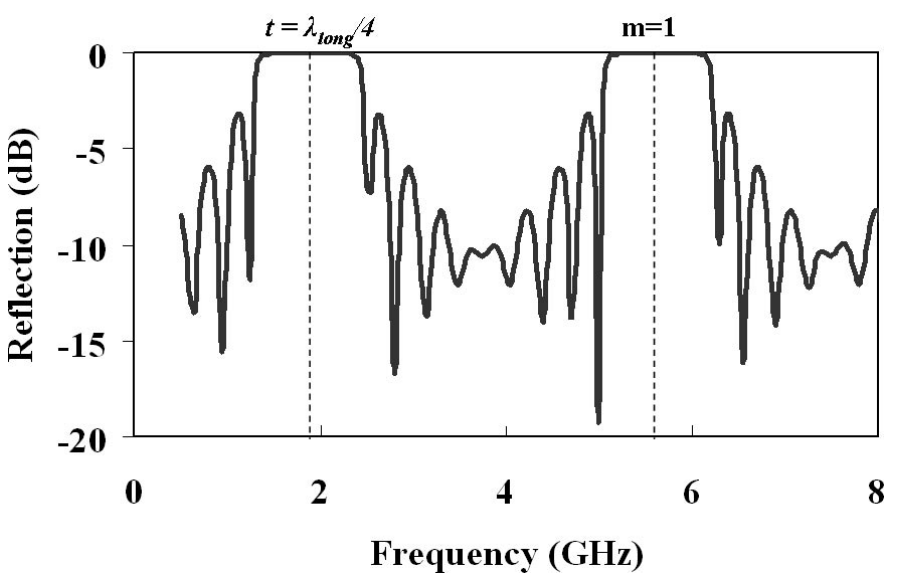

Fig. 8. Reflection spectra of the longitudinal waves in a nine-layer $\mathrm{Ta}_{2} \mathrm{O}_{5} /$ $\mathrm{SiO}_{2}$ quarter-wave reflector.

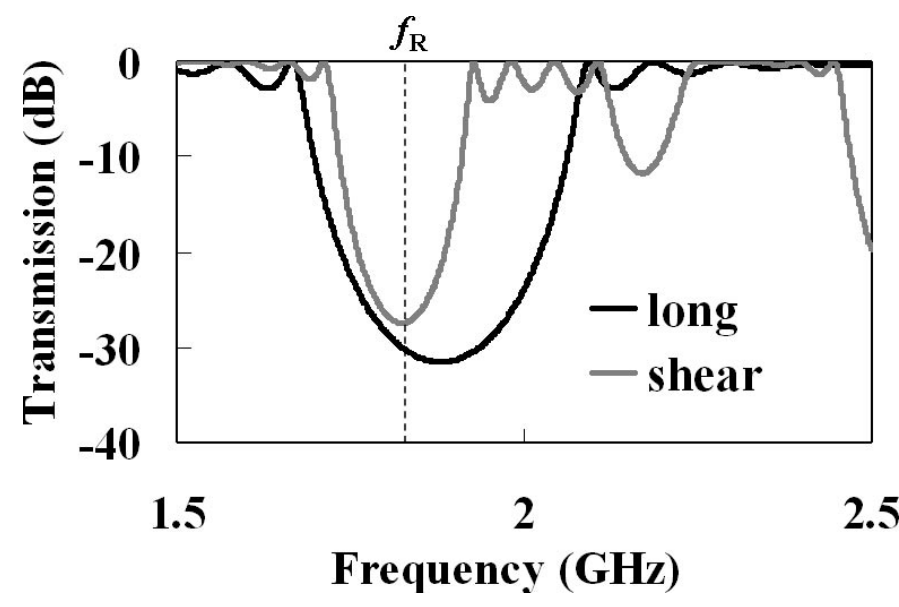

Fig. 9. Transmission curves of a nine-layer optimized reflector stack of $\mathrm{SiO}_{2} / \mathrm{Ta}_{2} \mathrm{O}_{5}$. The transmission of longitudinal waves at resonance is about $-31.1 \mathrm{~dB}$ nearing quarter-wave performance. The transmission of shear waves at resonance is about $-26.2 \mathrm{~dB}$.

From (27) it is deduced that two integers $m_{3}$ and $m_{4}$ are to be estimated that should satisfy the condition:

$$
m_{4}=K_{\mathrm{H}} \cdot m_{3}+\frac{\left(K_{\mathrm{H}}-1\right)}{2}
$$

where $K_{\mathrm{H}}=v_{\mathrm{H} \text {.long }} / v_{\mathrm{H} . \text { shear }}$. We have to obtain four integers $m_{1}, m_{2}, m_{3}$, and $m_{4}$ such that (26) and (28) are fulfilled. The values $m_{1}, m_{2}, m_{3}$, and $m_{4}$ are then plugged back into (24) and (27) to obtain the thicknesses of the low-impedance layer and high-impedance layer that efficiently reflect both longitudinal and shear waves. The thickness for a $\mathrm{SiO}_{2} / \mathrm{Ta}_{2} \mathrm{O}_{5}\left(m_{1}=1, m_{2}=1.96, m_{3}=\right.$ $1, m_{4}=2.2$ ) reflector stack is $2286 / 1764 \mathrm{~nm}$, calculated using this method with the described procedure. Fig. 9 shows the transmission curve of a nine-layer $\mathrm{SiO}_{2} / \mathrm{Ta}_{2} \mathrm{O}_{5}$ $(2286 \mathrm{~nm} / 1764 \mathrm{~nm})$ mirror optimized at $1.88 \mathrm{GHz}$ modeled in MathCAD. Note that the optimum for shear waves is not at the exact proper frequency because $m_{4}$ is then not an integer. 
TABLE III. Comparison of Three Approaches for a Nine-Layer $\mathrm{SiO}_{2} / \mathrm{TA}_{2} \mathrm{O}_{5}$ Stack at Resonant Frequency.

\begin{tabular}{lcc}
\hline Design approach & $T_{\text {long }}(\mathrm{dB})$ & $T_{\text {shear }}(\mathrm{dB})$ \\
\hline Quarter-wave stack & -31.5 & -0.59 \\
Stopband theory & -25.7 & -22.8 \\
General optimization & -27.4 & -17.3 \\
Diffraction grating & -31.1 & -26.2 \\
\hline
\end{tabular}

It may be hard to fulfill conditions (26) and (28) for the cases in which $K_{\mathrm{L}}$ and/or $K_{\mathrm{H}}$ are close to 2 . The righthand side tends to be fractional numbers for small integer values of $m_{1}$ and $m_{3}$ in (26) and (28), respectively. This means that for $K_{\mathrm{L}}=K_{\mathrm{H}}=2, m_{1}$ and $m_{3}$ need to be approximated to the nearest integer. If we use a reflector stack with $\mathrm{SiO}_{2}$ as low-impedance layer, $m_{1}=1, m_{2}=2$ fulfills (26) and an optimum at exact resonant frequency is expected. Some high-impedance materials will obey (28) better than others. It is concluded from the simulations that the diffraction grating method gives the best optimized stacks when $K_{\mathrm{L}}$ and $K_{\mathrm{H}}$ deviate from 2 by $15 \%$ or more. Nevertheless, the layer thicknesses obtained by diffraction grating approach are relatively thick compared with the stopband theory approach. A thickness optimization is possible for some stacks with high impedance contrast which is not discussed in this work.

\section{B. Comparison of Two Approaches}

Both of the approaches described in Sections III-A and III-B are based on velocity ratios. If the velocity ratios $\mathrm{K}_{\mathrm{L}}$ and $\mathrm{K}_{\mathrm{H}}$ are close to 2 , the reflector stack can be designed using the basic reflector design method (8) and can be implemented with the spacer configuration described recently [12]. In the cases in which a trade-off is to be found between longitudinal and shear transmission, the optimized thicknesses can be obtained by varying $c$ as described in the general optimization scheme described in Section III-A. The layer thicknesses calculated using the diffraction grating method described in Section III-B are generally greater than $1 \mu \mathrm{m}$ for the resonant frequency of $1.88 \mathrm{GHz}$ for the same number of layers. However, this method gives best results when the average velocity ratio [as described by (12)] deviates from the usual value of 2 by $15 \%$ or more. On the other hand, the stopband approach performs best when the average velocity ratio deviates from 2 by $15 \%$ or less, resulting in a phase error of less than $20 \%$. Hence, the diffraction grating approach complements the basic stopband approach. If there are no constraints for the fabrication of thicker layers, the diffraction grating method gives a straight forward guideline for the stack design.

Table III summarizes the comparison of transmission of longitudinal and shear waves at $f_{\mathrm{R}}$ in a nine-layer $\mathrm{SiO}_{2} /$ $\mathrm{Ta}_{2} \mathrm{O}_{5}$ acoustic mirror designed with three approaches. It can be concluded that the transmission of longitudinal waves is nearing quarter-wave performance for the SMR with stacks designed using diffraction grating method.

\section{Modeling And Simulation}

A 2-D FEM physical model of the $\mathrm{SMR}$ with $\mathrm{SiO}_{2} /$ $\mathrm{Ta}_{2} \mathrm{O}_{5}$ stacks designed using both the approaches were developed using the Comsol Multiphysics tool (Comsol $\mathrm{AB}$, Stockholm, Sweden) to verify the design theories. As the average velocity ratio as described in (12) is close to 2 in this material combination, (8) will hold well in this case. The optimized thicknesses were implemented in the spacer configuration described in [12]. Stacks designed using diffraction grating approach (Section III-B) were also simulated and the displacement profiles were compared. Half-structure simulations were done, exploiting symmetry with continuous layers for reflector and bottom electrode. The discontinuous layers were the top electrode and the piezoelectric layer (with free edge boundaries). In simulations, at the right-hand side, the $U_{x}=U_{z}=0$ boundary condition was applied and at the left-hand side, the symmetric boundary condition $\left(U_{x}=0, \partial U_{z} / \partial x=0\right)$ was applied. Fig. 10 shows the simulation results of the displacement profile of longitudinal and shear waves at resonant frequency in an SMR with (a) a quarter-wave stack, (b) and (c) shear-optimized stacks designed using the theory described in Section III-A, and (d) a shear-optimized stack designed using the diffraction grating method (Section IIIB). This is an illustration of the effect of the stack obtained with all proposed design approaches. It is evident from the figures that in the resonator with conventional quarter-wave reflector, shear waves are leaking into the substrate, whereas in the case of shear-optimized stacks, leakage of shear waves into the substrate is suppressed.

\section{Experimental Results}

SMRs with optimized reflector stacks have been realized and characterized at NXP Semiconductors, Nijmegen, The Netherlands. Some of them closely resemble the stacks (see Fig. 3) proposed by the stopband theory with spacer layers [7], [8]. The most noticeable deviation is the topmost low-impedance layer for optimizing the dispersion curve, which is not discussed in this work. The electrical responses of the SMRs were analyzed by RF measurements using a ZVB20 vector network analyzer (Rohde \& Schwarz, Munich, Germany). 1-port scattering parameters $\left(S_{11}\right)$ were measured using GSG-250 coplanar RF probes (Cascade Microtech, Beaverton, OR) and impedance curves were plotted. A low power level of $-20 \mathrm{dBm}$ was used to avoid any nonlinearities in the measurements. Very small frequency steps of $25 \mathrm{kHz}$ were used to extract the $Q$-factor from the steepness of the phase $(\varphi(f))$ curves (measured directly from the analyzer) as [13]

$$
Q_{\mathrm{A}}=\left.0.5 \cdot f_{p} \cdot \frac{d \phi(f)}{d f}\right|_{f=f_{\mathrm{A}}},
$$

where $Q_{\mathrm{A}}$ is the $Q$ factor at anti-resonance and $f_{\mathrm{A}}$ is the anti-resonance frequency. Although the $Q$ values calcu- 


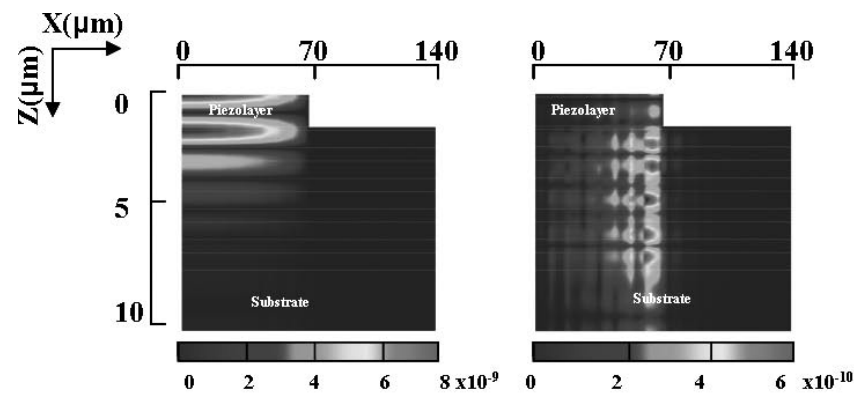

(a)
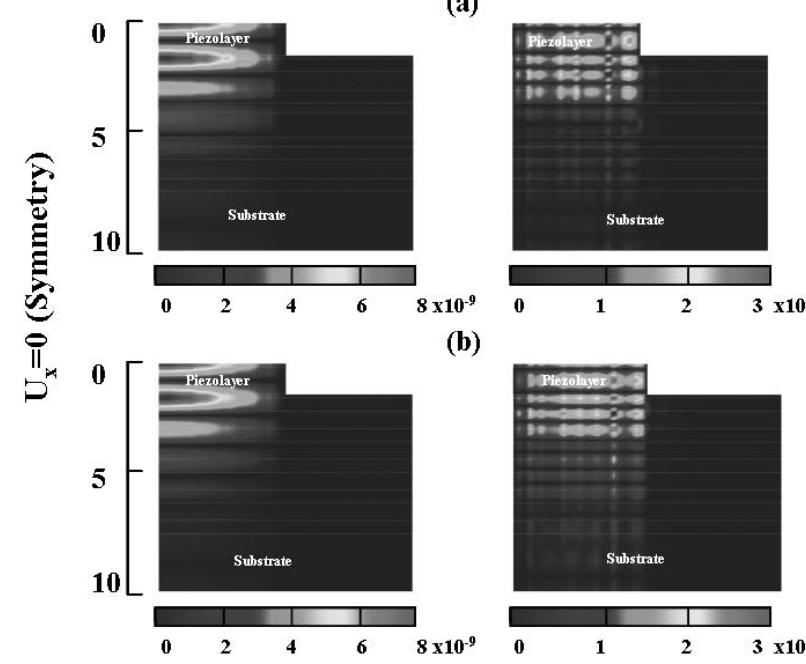

(b)

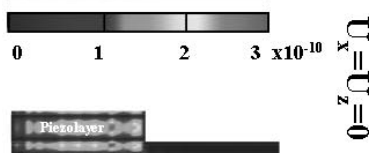

(c)
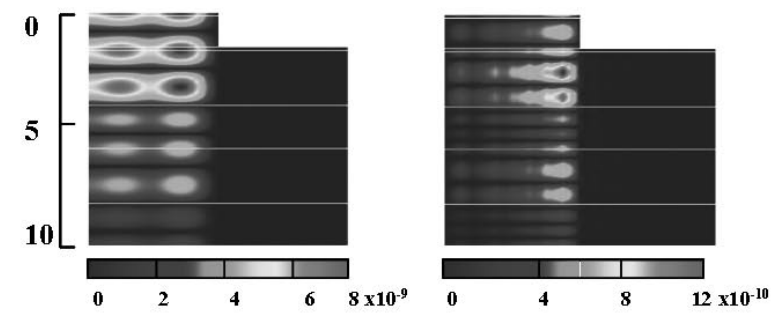

Bottom :Free

(d)

Fig. 10. 2-D FEM results for displacement of longitudinal waves (left) and shear waves (right) at resonant frequency in an SMR with $\mathrm{SiO}_{2}$ / $\mathrm{Ta}_{2} \mathrm{O}_{5}$ reflector stack using (a) a quarter-wave stack $(762 \mathrm{~nm} / 588 \mathrm{~nm})$, (b) shear-optimized stack using stopband theory, $c=1.73$, RS:(559/862/ 559/431/1118/431/559/862/559 (nm), Spacer: 431/1118/431), (c) shear optimized stack using optimization scheme, $c=1.7(617 \mathrm{~nm} / 810 \mathrm{~nm})$, and (d) shear optimized stack using diffraction $\mathrm{g}$ rating approach $(2286 \mathrm{~nm} / 1764 \mathrm{~nm})$ in a nine-layer $\mathrm{SiO}_{2} / \mathrm{Ta}_{2} \mathrm{O}_{5}$ reflector stack. Plane of symmetry is defined in the left edge of the device. Note that in (d), results are shown only for $10 \mu \mathrm{m}$ for comparison; the simulation is for 9 layers.

lated by this method are sensitive to the frequency step $d f$ [5], for an illustration of the efficiency of the reflector stack, this method is used here.

Fig. 11 shows a comparison of the measured and FEM simulated impedance curves for a resonator designed with conventional quarter-wave mirror and the optimized reflector for dual-wave reflection; the simulations are in good agreement with the measurements. In SMR with a shear-optimized reflector stack, the thickness of the piezoelectric layer was adapted to bring $f_{\mathrm{R}}$ to $1.88 \mathrm{GHz}$. For a

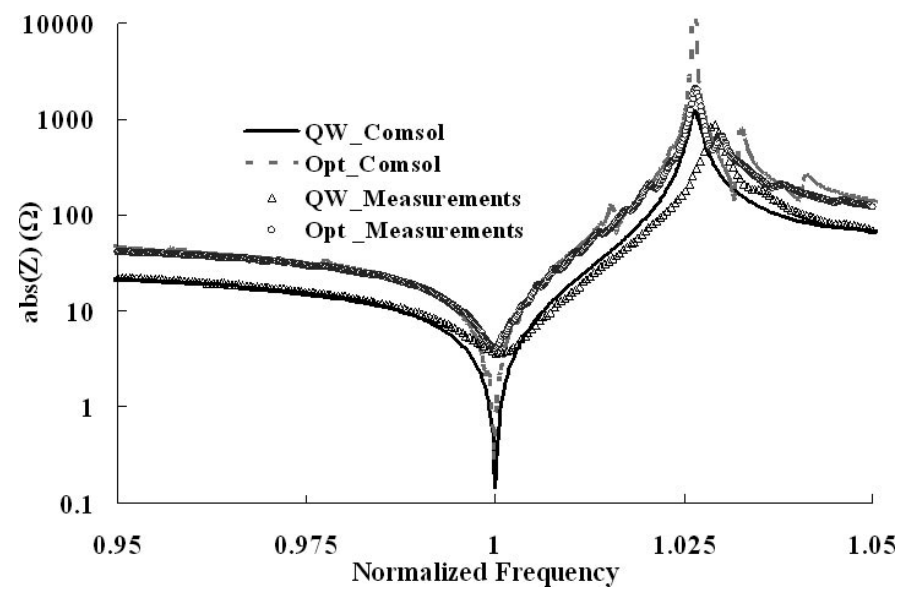

Fig. 11. Comparison of impedance curves from Comsol model and the measurements in an SMR with quarter-wave $(\mathrm{QW})$ reflector stack and shear-optimized (Opt) reflector stack.

$300 \times 100 \mu \mathrm{m}^{2}$ conventional quarter-wave mirror device, a $Q_{\mathrm{A}}$ value of 1075 is obtained, whereas the device with optimized stacks exhibits a $Q_{\mathrm{A}}$ of around 1940. Because $Q_{\mathrm{R}}$ at resonance is limited by ohmic rather than acoustic losses, the improvement in $Q$ at resonance, $Q_{\mathrm{R}}$ seems to be small. Note that the shear-optimized mirror increased the maximum impedance at anti-resonance by more than a factor of 2 compared with the quarter-wave stack, because of the different piezoelectric layer thicknesses among the devices. Furthermore, impedance plots of shear-optimized reflector stack obtained from Comsol simulations and measurements shown in Fig. 11 show spurious resonance peaks besides the main peak; the appearance of spurs is a sign of energy confinement. Although this is an undesired side effect, this is an indication that the acoustic energy is better confined in the new reflector than in the conventional quarter-wavelength reflector.

However, these spurs make a proper extraction of a quality factor difficult. To extract the substrate losses, we should measure the impedance curve of a very large resonator (unlike the small dimension of $300 \times 100 \mu \mathrm{m}^{2}$ ) or, even better, plot $1 / Q_{\mathrm{A}}$ versus perimeter/area [8]. Fig. 12 shows the scaling of quality factor for resonators with quarter-wave stack, shear-optimized stack with and without a frame region. The spread on the $1 / Q_{\mathrm{A}}$ is much larger for shear-optimized stacks than for quarter-wave stacks, again because spurious modes make good extraction of $Q$ difficult. We see that the shear-optimized reflector alone does not give an improvement in $Q_{\mathrm{A}}$, for large or for small resonators. This is because the fraction of stored acoustic energy in the shear waves is small, so an improvement of shear-wave reflectivity has little impact on the overall $Q_{\mathrm{A}}$. Small resonators have more energy in shear waves but their main loss mechanism is sideways acoustic radiation rather than loss into the substrate.

The improvement in $Q$ factor can be seen when the frame region is applied for the devices with shear optimized stacks. The combination of shear-optimized stacks with a frame region is a known method to decrease loss 


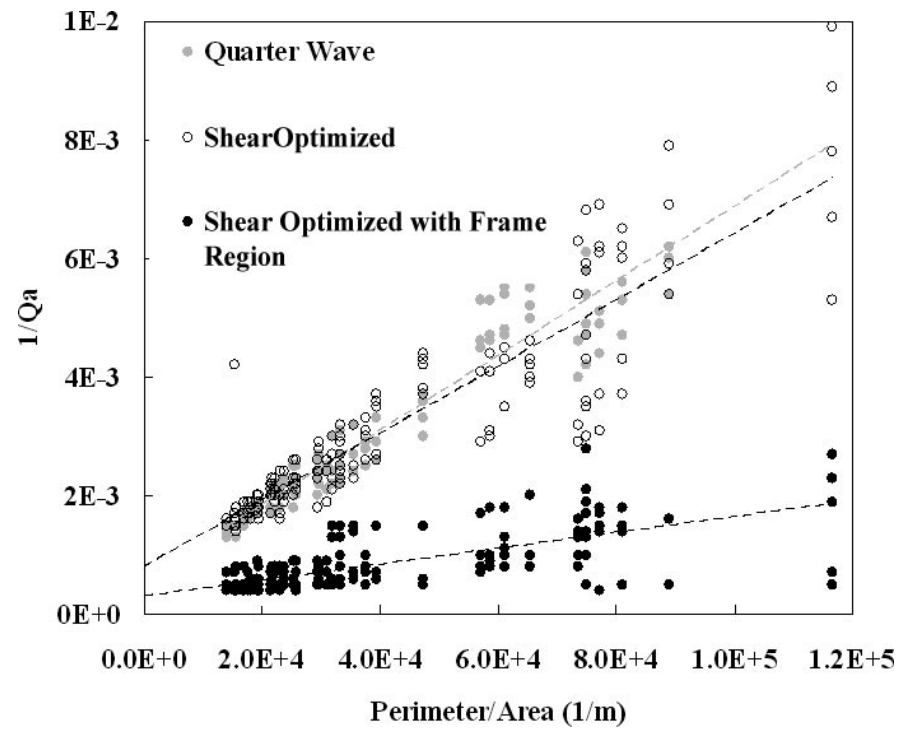

Fig. 12. Experimental data showing scaling of quality factor at antiresonance, $Q_{\mathrm{A}}$, for resonators with quarter-wave $(\mathrm{QW})$, shear-optimized stack with and without a frame region [7]. The 1-D or extrapolated value at the vertical axis gives us $1 / Q_{\mathrm{A}}$ of the active device (corresponding to $Q_{\mathrm{A}}=1600$ for $\mathrm{QW}$ and $Q_{\mathrm{A}}=3300$ for optimized stack); the slope of this (scattered) curve tells us about the parasitic $Q_{\mathrm{A}}$ caused by perimeter/ edge effects [7], [8].

caused by lateral radiation and thus to improve $Q_{A}$ for small devices [11]. However, for a frame-region device to work, shear wave reflection is a necessary condition [9]. The scattering of the graph is strongly reduced by applying the frame region in [7]. Optimizing the reflector stack for energy confinement thus also allows a straightforward use of the frame region. The extracted 1-D quality factor for a resonator with optimized stack and the frame region is around 3300 and, even for small devices, $Q_{\mathrm{A}}$ well above 1000 are found. Fig. 12 thus shows that shear wave reflection is reached and demonstrates the effectiveness of the proposed optimization scheme.

\section{CONCLUSiON}

Design approaches derived from optics have been presented for optimizing dual reflection of longitudinal and shear waves in solidly mounted BAW resonators. Two design approaches are discussed, one based on stopband theory and the other based on the diffraction grating method, both involving the velocity ratio of longitudinal to shear wave in the reflector stack layers. A novel optimization scheme is presented that allows the designer to find a trade-off between longitudinal and shear reflection in the stack. The procedure has been demonstrated for different material combinations, and in all the cases a minimum transmission of $-25 \mathrm{~dB}$ and $-20 \mathrm{~dB}$ at resonance frequencies were obtained for longitudinal and shear waves, respectively. The results obtained from the design approaches have been implemented in FEM simulations to verify the theories. SMRs employing an optimized reflector stack have been characterized and the measurements show good agreement with FEM simulations. The adaptability of these approaches makes it a potential design guideline for devices with minimized acoustic losses into the substrate. Experimental results demonstrate resonators working around $1.88 \mathrm{GHz}$ with improved $Q$ values as high as 3300 .

\section{ACKNOWLEDGMent}

The authors thank the former BAW team of NXP Research, Eindhoven, The Netherlands, especially Dr. J. Ruigrok, for the valuable Comsol discussions. The authors thank Dr. R. M. de Ridder (MESA+, University of Twente, The Netherlands) for stimulating discussions on the diffraction grating approach. Special thanks are given to H. Bosch and J.-W. Lobeek from NXP Semiconductors, Nijmegen, for the fabricated devices. Thanks are also due to H. de Vries, University of Twente, The Netherlands, for helping with RF measurements.

\section{REFERENCES}

[1] H. P. Loebl, C. Metzmacher, R. F. Milsom, P. Lok, F. Van Straten, and A. Tuinhout, "RF bulk acoustic resonators and filters," J. Electroceram., vol. 12, pp. 109-118, 2004.

[2] K. M. Lakin, J. R. Belsick, J. P. McDonald, K. T. McCarron, and C. W. Andrus, "Bulk acoustic wave resonators and filters for applications above $2 \mathrm{GHz}$," in IEEE MTT-S Int. Symp. Dig., Jun. 2002, pp. 1487-1490.

[3] E. Schmidhammer, B. Bader, W. Sauer, M. Schmiedgen, H. Heinze, C. Eggs, and T. Metzger, "Design flow and methodology on the design of BAW components," in IEEE MTT-S Int. Symp. Dig., Jun. 2005, pp. 233-236.

[4] K. M. Lakin, G. R. Kline, and K. T. McCarron, "High-Q microwave acoustic resonators and filters," IEEE Trans. Microw. Theory Tech., vol. 41, no. 12, pp. 2139-2146, 1993.

[5] F. Z. Bi and B. P. Barber, "Bulk acoustic wave RF technology," IEEE Microw. Mag., vol. 9, no. 5, pp. 65-80, Oct. 2008.

[6] R. Aigner, "Bringing BAW technology into volume production: The ten commandments and the seven deadly sins," in IEEE Int. Symp. Acoustic Wave Devices for Future Mobile Communication Systems, 2007, pp. 85-91.

[7] J. W. Lobeek, R. Strijbos, A. B. M. Jansman, N. X. Li, A. B. Smolders, and N. Pulsford, "High-Q BAW resonator on Pt/ $\mathrm{Ta}_{2} \mathrm{O}_{5} /$ $\mathrm{SiO}_{2}$-based reflector stack," in Proc. IEEE Microwave Symp., 2007, pp. 2047-2050.

[8] R. Strijbos, A. B. M. Jansman, J. W. Lobeek, N. X. Li, and N. Pulsford, "Design and characterization of high-Q solidly-mounted bulk acoustic wave filters," in Proc. IEEE Electronic Components and Technology Conf., 2007, pp. 169-174.

[9] G. G. Fattinger, S. Marksteiner, J. Kaitila, and R. Aigner, "Optimization of acoustic dispersion for high performance thin film BAW resonators," Proc. IEEE Ultrasonics Symp., pp. 1175-1178, 2005.

[10] S. Marksteiner, G. G. Fattinger, R. Aigner, and J. Kaitila, "Acoustic reflector for a BAW resonator providing specified reflection of both shear wave and longitudinal waves," U.S. Patent 006933807B2, Aug. $23,2005$.

[11] J. Kaitila, "Review of wave propagation in BAW thin film devices progress and prospects," Proc. IEEE Ultrasonics Symp., 2007, pp. 120-129.

[12] S. Jose, A. B. M. Jansman, and R. J. E. Hueting, "A design procedure for an acoustic mirror providing dual reflection of longitudinal and shear waves in solidly mounted BAW resonators (SMRs)," in Proc. IEEE Ultrasonics Symp., Sep. 2009, pp. 2111-2114.

[13] S. Marksteiner, J. Kaitila, G. G. Fattinger, and R. Aigner, "Optimization of acoustic mirrors for solidly mounted BAW resonators," in Proc. IEEE Ultrasonics Symp., 2005, pp. 329-332. 
[14] Z. Knittl, Optics of Thin Films, 1st ed., New York, NY: Wiley, 1976. pp. 66-67, 86-103.

[15] Lord Rayleigh, "On the reflection of light from a regularly stratified medium," Proc. Roy. Soc. London A, vol. 93, no. 655, pp. 565-577, 1917.

[16] G. Pei-fu and Z. Zhen-rong, "Design of non-polarizing thin film edge filters," J. Zhejiang Univ. Sci. A, vol. 7, no. 6, pp. 1037-1040, 2006.

[17] A. Thelen, Design of Optical Interference Coatings. ser. Optical and Electro-Optical Engineering, New York, NY: McGraw-Hill, 1988.

[18] S. G. Alekseev, Yu. V. Gulyaev, G. D. Mansfeld, and V. I. Pustovoit, "Propagation of acoustic waves through periodic structures containing phase non-regularities," in IEEE Proc. Ultrasonics Symp., 2005, pp. 2124-2127.

[19] E. Hecht, Optics, 3rd ed., Reading, MA: Addison Wesley Longman, 1998 , pp. $422,487-494$

[20] F. A. Jenkins and H. E.White, Fundamentals of Optics, 4th ed., New York, NY: McGraw-Hill, 1981, pp. 355-356, 380.

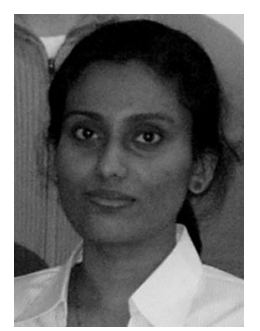

Sumy Jose was born in Kerala, India, in 1982. She received her bachelor's degree from MG University (Kerala) in physics in 2002. She graduated in applied electronics from the National Institute of Technology, Tiruchirappally, India, in 2004. She joined as a Project Assistant at the Indian Institute of Science, Bangalore, in 2005 where she has been involved in research projects in the area of RF MEMS modeling. Currently, she is working towards a Ph.D. degree at the Semiconductor Components group of University of Twente, The Netherlands, on BAW modeling. Her primary research interests include physics-based multi-domain modeling and design of acoustic and MEMS devices.

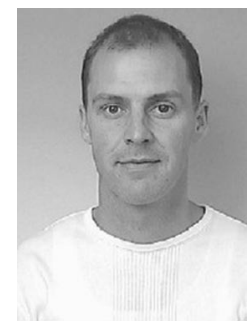

André Jansman was born in Raalte, The Netherlands, in 1971. He received the M.S. degree in applied phyics (1995) and his Ph.D. degree (1999) at the University of Twente, Enschede, The Netherlands. In 2000, he joined Philips Research Laboratories, later NXP Research, Eindhoven, The Netherlands, where he has been working on thinfilm technologies for the realization of high-quality integrated RF passive, bulk-acoustic wave filters. Currently he works on model aspects of magnetoresistive sensors for automotive applications.

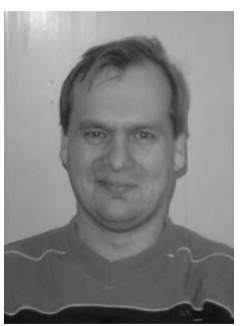

Raymond J. E. Hueting (S'94-M'98-SM'06) was born in Bussum, The Netherlands, on May 28, 1968. He obtained his M.Sc. degree (cum laude) and Ph.D. degree in electrical engineering from Delft University of Technology in 1992 and 1997, respectively. His Ph.D. thesis dealt with the device physics of SiGe-based heterojunction bipolar transistors.

In 1997, he joined Philips semiconductors, Nijmegen, The Netherlands, where he worked on lateral power MOSFETs in SOI-based BCD-IC processes used for automotive and audio applications. He joined Philips research laboratories in Eindhoven, The Netherlands in 1998, and in Leuven, Belgium, in 2001, where he worked on device physics of trench-gate power MOSFETs, used for power supplies and automotive applications, and was involved in the development of novel silicon devices, including SiGe-based heterojunction devices. Since December 2004, he has been working at the electrical engineering department of the University of Twente, The Netherlands, in the field of semiconductor device physics and modeling.

Dr. Raymond J. E. Hueting authored and co-authored more than 30 papers and more than 60 patents including 33 U.S. patents. He participates and participated in the technical program committees of the ESSDERC and the ISPSD conference, respectively.

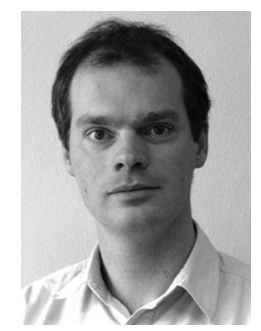

Jurriaan Schmitz (M '02-SM '06) received his M.Sc. cum laude and Ph.D. degrees in experimental physics at the University of Amsterdam in 1990 and 1994, respectively. He then joined Philips Research as a Senior Scientist, studying CMOS transistor scaling, characterization, and reliability. Since 2002, he has been a full professor at the University of Twente, leading the Group of Semiconductor Components. He serves as a TPC member of the IEDM, ESSDERC, and ICMTS conferences, and will be General Chairman of the ICMTS conference in 2011. Prof. Schmitz authored or co-authored more than 170 journal and conference papers and holds 16 US patents. His research interests include CMOS post-processing and device characterization. 